\title{
Philosophiques
}

\section{René Girard, Shakespeare : les feux de l'envie, tr. fr. de Bernard Vincent, Paris, Grasset, 1990.}

\section{Pierre Gravel}

Volume 20, numéro 2, automne 1993

Perspectives sur la phénoménologie et l’intentionnalité

URI : https://id.erudit.org/iderudit/027244ar

DOI : https://doi.org/10.7202/027244ar

Aller au sommaire du numéro

Éditeur(s)

Société de philosophie du Québec

ISSN

0316-2923 (imprimé)

1492-1391 (numérique)

Découvrir la revue

Citer ce compte rendu

Gravel, P. (1993). Compte rendu de [René Girard, Shakespeare : les feux de l'envie, tr. fr. de Bernard Vincent, Paris, Grasset, 1990.] Philosophiques, 20(2), 516-518. https://doi.org/10.7202/027244ar d'utilisation que vous pouvez consulter en ligne.

https://apropos.erudit.org/fr/usagers/politique-dutilisation/ 
René Girard, Shakespeare : les feux de l'envie, tr. fr. de Bernard Vincent, Paris, Grasset, 1990.

par Pierre Gravel

Il s'agit sans aucun doute du meilleur des sept livres que nous a donnés René Girard depuis ig6I, année de la publication de Mensonge romantique et vérité romanesque, son premier livre. Je tiens à souligner ce texte puisque depuis cette date, René Girard le rappellera d'ailleurs lui-même (p. 58, par exemple), une méthode d'interprétation s'était affirmée qui n'a cessé de se consolider, de s'étendre et, disons-le, de se répéter. On aurait pu s'attendre à une lente évolution, voire à une maturation ou une transformation, mais non, la méthode, 
fondée essentiellement sur la notion de désir mimétique et de notions connexes comme celle du double ou du « double bind » empruntée à Freud, la méthode est donnée et conquise dès le début. A l'origine donc, et il s'agit d'une théorie de l'origine : le désir mimétique engendre en effet la crise sacrificielle, laquelle se résout enfin par la mécanique du bouc émissaire. Jules César, on le devine, sera bientôt convoqué. Le cas est suffisamment rare pour le souligner : dès le début la théorie est complète, les principaux éléments en sont dégagés et présentés sous forme épurée. Notons par ailleurs que la méthode d'analyse littéraire déborde considérablement le domaine relativement clos des études littéraires pour s'attaquer aux diverses anthropologies (Lévi Strauss principalement), à la psychanalyse (voir la charge contre le supposé complexe d'cEdipe que nous avait servi La violence et le sacré, 1972), aux sciences humaines en général, mais aussi à la religion dans sa forme chrétienne (voir Des choses cachées depuis la fondation dumonde, 1978). Cela pourra paraître lassant, mais c'est un fait: au lieu de se transformer ou d'évoluer, la puissance interprétative, car la théorie est puissante, s'étend cette fois à Shakespeare et à une bonne partie de son œuvre.

Vingt-deux pièces sont en effet partiellement étudiées, sans compter quelques sonnets et, mais pourquoi pas, une partie de l'Ulysse de James Joyce qui se rapporte à Shakespeare. Vingt-deux pièces, dans ce fort volume de 437 pages grand format, cela signifie que R. G. ne tient aucun compte de la distinction et de la présentation des œuvres en genres, ces genres canoniques que furent les comédies, drames historiques, tragédies et sonnets. On peut le regretter : pourquoi shakespeare, en effet, aurait-il développé ou employé les mêmes opérateurs dans les divers genres où il a par ailleurs excellé ? C'est, on peut le souligner à cette occasion, que la méthode de R. G. est globalisante, sinon totalisante. Mais il y a, à notre avis, pire : tout se passe dans la stratégie de lecture qui nous est proposée, comme si shakespeare avait non seulement lu R. G., et je n'exagère aucunement, mais avait construit son œuvre à partir des thèses de notre auteur, ce qui englobe aussi l'Ulysse de James Joyce (voir p. 3 iz et $s q$ )!

Une telle affirmation pourra paraître grosse, sinon énorme, donnons quelques illustrations : en p. 25 il est question de la « source proprement shakespearienne d'intelligibilité, je veux dire le désir mimétique »; p. 56, à propos des implications de la théorie du double sur laquelle $\mathrm{R}$. G. insiste avec raison, il ne craint pas d'écrire : « Shakespeare découvre peu à peu les implications de sa propre vision des choses » (souligné par nous) ; en p. 59, à propos d'une analyse de deux passages des Deux gentilshommes de Vérone, il ajoute, ce qui relève sans aucun doute d'un puissant savoir : « Il a écrit cette scène à un moment crucial de sa propre assimilation du processus mimétique »; ce qui n'empêchera pas notre auteur de reconnaître, en page $3 \mathbf{I}$ par exemple, qu'il a quelque peu forcé les choses : « pour les besoins de la démonstration, j'ai quelque peu forcé la symétrie mimétique des deux protagonistes », etc. ! Remarquons enfin que R. G. a consacré pas moins de cinq chapitres à l'analyse, centrale à notre avis dans ce livre, de Jules César, centrale parce que la théorie de R. G. s'y voit complètement développée, du désir mimétique au meurtre fondateur.

Il s'agit en quelque sorte d'un cas classique dans le domaine des études littéraires, ou disons mieux : dans le domaine de l'esthétique de la littérature, 
cas classique qui peut se résumer en une alternative : ou bien l'œuvre littéraire donne à penser pour reprendre l'expression de Paul Eluard, et alors, comme puissance énigmatique, elle peut nous inviter à remettre en question les instruments théoriques que nous mettons en œuvre à son propos, ou bien le texte devient littéralement prétexte et ne vaut que comme illustration en quelque sorte de la valeur de la méthode qui gagne et réaffirme sa vérité, de son application au plus grand nombre possible de cas et de genres singuliers. Les différents structuralismes particulièrement à la mode dans les années 60 en forment l'exemple le plus parfait : sémiologie, psychanalyse, un certain marxisme, etc. Disons que dans le cas présent, $R$. G. se tient à la limite de ces deux attitudes ici trop rapidement décrites. Il s'agit sans contredit de son meilleur livre, celui, du moins, où la méthode est le plus pédagogiquement développée, mais Shakespeare reste à relire dans la structure interne même où chacune de ses œuvres nous est présentee, dans la puissance énigmatique qui est la sienne. Shakespeare peut encore donner à penser pour tous ceux, bien évidemment, qui sauront rester à l'écoute de son thêâtre en négligeant activement d'en faire le prétexte muet d'une construction savante par ailleurs toujours possible.

\section{Département de philosophie}

Université de Montréal 